\title{
Texture Recognition using Hybrid Fractal and Blocking Approach
}

\author{
Salah S. AL-Rawi, Ph.D \\ Computer Science Department \\ College of Computer \\ University of Anbar, Ramadi, Iraq
}

\author{
Ahmed Tarik, Ph.D \\ Computer Science Department \\ College of University of Technology \\ Baghdad , Iraq
}

\author{
Eman Turky mahdi \\ Computer Science Department \\ College of Computer \\ University of Anbar, Ramadi, Iraq
}

\begin{abstract}
Texture analysis is an important and useful area of study in machine vision. Most natural surfaces exhibit texture and a successful vision system must be able to deal with such like surfaces. Many natural surfaces have a statistical quality of roughness and self-similarity at different scales. Fractals are very useful and have become popular in modeling these properties in image processing. This work adopts analyzing samples by three methods fractal dimension, block approach and Hybrid method (fractal dimension method with block approach). The fractal dimension get a highest recognition rate among remaining used methods, it obtain a rate $95 \%$ as compare with $40 \%$ Block Approach model, $65.5 \%$ Hybrid method. The results show the efficiency of fractal dimension recognition than blocking approach recognition and hybrid recognition in textures.
\end{abstract}

\section{General Terms}

Image Processing, Texture Recognition and Binarization method.

\section{Keywords}

Fractal Dimension, Box-Counting Method and Blocking Approach.

\section{INTRODUCTION}

Texture analysis is one of the modern techniques used in analyzing and classifying the objects of images presenting repetition of fundamental image elements, and it is a basic issue in image processing and computer vision [1,2]. The categories texture analysis techniques can be represented into four approaches: statistical approaches, structural approaches, filter based approaches, and model based approaches [3].Model based approach, among many others; represent autoregressive models, random field models, the epitome model, and the fractal models [4]. The concept of the fractals model, as an indicator of surface roughness, was first introduced by Mandelbrot [5]. Fractal theory has been widely applied to different scientific areas, particularly in image processing [6]. According to their surface Rough surfaces certainly appear more frequently than smooth surfaces in nature [Mandelbrot, 1982], but are also common in synthetic environments, such as fabrics, carpeting and in the microstructured texture found on otherwise flat surfaces. Rough surfaces are exemplary modeled by using the tools of fractal geometry [1]. Therefore the fractal functions are considered a good choice to represent natural tissue surfaces which were chosen because of the importance of short period characteristics of classifying images since the fractal dimension of a surface has an approximation of complete relation with surface toughness [7]. Texture configuration is one of the most important characteristics used in identifying objects or regions of interests in an image because machine vision and many task such as surface inspection. Scene classification, and surface orientation and shape determination. For example, layer surface of texture features are used in the inspection of semiconductor wafers, gray-level distribution features of homogeneous textured regions are used in the classification of aerial imagery, and variations in texture patterns due to perspective projection are used to determine three-dimensional shapes of objects [8]. Hence , texture is measure of the surface roughness ,another term without an accepted or universal quantitative meaning[9]. In the gray levels of texture characterized by spatial distribution of a neighborhood spaces, thus, texture cannot be defined for appoint ,but as repeating pattern of local variation in image intensity ;some of studies describe the texture as "thing $\mathrm{s}$ arranged in a pattern ".texture are a function of things and pattern mathematically [10]. Some research based on the Bouligand-Minkowski predictors; dividing the original image recursively into equal parts and estimate the average and the deviation of the latest predictors computed over each part it achieves better results than classical and state-of-the-art texture descriptors, such as Local Binary Patterns, Gaborwavelets and co-occurrence matrix[11]. Some other research, implement an algorithm that provide more than one slide for the image based on new method develop multithresolding and then extract feature of fractal dimension using Box Counting Method for each slide[12].

\section{PREVIOUS WORKS}

Akram Alsadat and Saeed Mozaffari, 2011: This paper proposed a new method based on fractal geometry for Farsi/Arabic font recognition. The main features are obtained by combining the BCD, DCD, and DLA techniques. Dataset includes 2000 samples of 10 typefaces, each containing four sizes. The average recognition rates obtained for these 10 fonts and 4 sizes ( 40 classes) using RBF and KNN classifiers are $96 \%$ and $91 \%$ respectively. Experimental results show that this algorithm is robust against skew[13].

Azmi Tawfik Alrawi and ect, 2012:In this paper focus on implement novel supervised algorithm for multitexture segmentation and this algorithm based on blocking procedure where each image divide into block and extract vector feature for each block to classification these block based on these feature. These feature extract using Box Counting Method $(\mathrm{BCM})$. The implemented algorithm provide more than one slide for the image based on new method produce multithresolding, after this use BCM to generate single feature for each slide[12]. 
Ji-xiang Du and ect, 2012: This paper proposed a new method of describing the characteristics of plant leaves based on the outline fractal dimension and venation fractal dimension. First, using multiple threshold edge detection method to separate leaf edge and vein, and get multiple veins. Then, the two-dimensional fractal dimension of the leaf edge image and multiple vein images will be calculated, and a new ring projection wavelet fractal feature for leaf shape is also adopted. Finally, these two kinds' fractal dimension features are used to plant leaves classification and recognition. The experimental results demonstrated the effectiveness of the fractal dimension feature method[13].

\section{FRACTAL GEOMETRY}

It is a mathematical science that studies the properties and behavior of fractals. The object description in many situations cannot be explained easily by classical geometry, and it has often been applied in science, technology, and computergenerated art. The conceptual roots of fractals can be traced to measure the size of objects for which traditional definitions based on Euclidean geometry or calculus fail. The scientist efforts devoted with success by declaring, first announce mathematical method which called fractal geometry (Mandelbrot 1970). Principle of fractal geometry is the concept of self-similarity, in which an object appears to look similar at different scales. This concept can be applied to systems of varying physical size, relying on the complexity and diversity of the fractal model that is considered [15]. Fractal objects appeared in nature as coastlines, snowflakes, fern trees, bacteria, clouds and mountains. Some of the most important properties of fractals are self-similarity as described before, chaos, and they have non-integer dimension, which gives a quantitative measure of self-similarity and scaling. Fractal geometry is not concerned with the explicit shape of objects. Instead, fractal geometry identifies the value that quantifies the shape of the object's surface, the fractal dimension of the object.

\section{FRACTAL DIMENSION}

A basic consideration in the fractal characteristic measurements which it use for description and classification fractal processes, is their fractal dimension (FD). The fractal dimension provides an objective means of quantifying the fractal property of an object and comparing objects observed in the natural world [16]. Fractal dimension is a familiar parameter for explaining certain phenomena and for describing natural textures and it represents an important feature of textural images. Hence, it is used to characterize roughness and self-similarity in a picture. This feature is used in texture description and classification, shape analysis and other computer vision and image processing problems [17]. Dimension is at the heart of all fractal geometry, which provides a reasonable basis for an invariant between different fractal objects. There are also experimental techniques capable of calculating the dimension of a given object, Based on the type of the shape or structure are dealing with nodes, lines, boundaries, curves, surfaces, spaces, etc.[18] .

\section{BOX-COUNTING METHOD (BCM)}

Box counting dimension consider as one of the most commonly use in fractal dimensions measurements. It's more popular ways because of the relatively simple concepts in the mathematical calculation and estimation. To estimate the $\mathrm{BCD}$, according to equation (2), first step we need to calculate the number of boxes $A(s)$ for entire block to solve overlap problem related to the calculation of FD for these blocks. The fractal dimension is obtained using the following equations: [12]

$\mathrm{H}=\frac{\sum_{s=0}^{k-2} \log (A(s)) * \log (N(s))}{\sum_{s=0}^{k-2} \log (A(s))^{\wedge}}$.

Where $\mathrm{H}$ is slop, $\mathrm{A}(\mathrm{s})$ is total number of boxes to all block, $\mathrm{N}(\mathrm{s})$ is number of box cover the structure, $\mathrm{k}$ is highest box size and $\mathrm{s}$ is box size. The FD of a curve can be ranging between 1 (which is equal to its topological dimension) and 2 (which is equal to the topological dimension of the plane that it can occupy).

\section{BLOCKING APPROACH ANALYZING}

The suggested model, in this approach, describe the texture image according to the spatial distributions of its object pixel's.by segmented binary image texture in to small windows $2 \mathrm{X} 2$ pixels and adequate these small windows with some given models to pic information about object of image texture distributions.

Topology models classified into Full object, Triple1 object, Triple2 object, Triple 3 object, Triple4 object, Horizental 1 object, Horizental 2 object, Vertical1 object, Vertical2 object, Main diagonal object and Second diagonal object. These models are described in figure (1). After classifying texture image windows according types of topology models, statistical repetition of each models are used as features of texture topology.

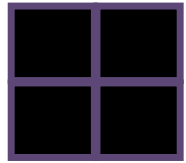

Full object

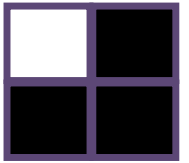

Triple1 object

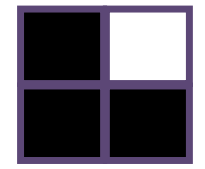

Triple2 object

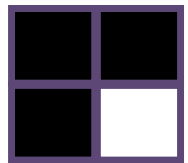

Triple3 object

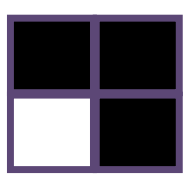

Triple4 object

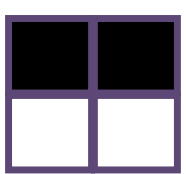

Horizental1 object

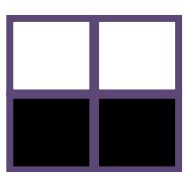

Horizental2 object

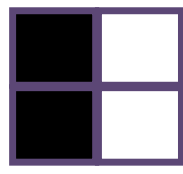

Vertical1 object

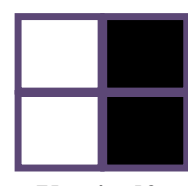

Vertical2 object

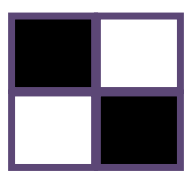

Main diagonal object

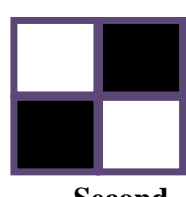

diagonal object
Figure (1): Block model types

\section{PROPOSED SYSTEM}

In this work, proposed system makes comparison between two different textures analyzing methods Box-counting method to calculate fractal code for texture image and blocking approach for texture topology, following figure (2) shows diagram of proposed system 


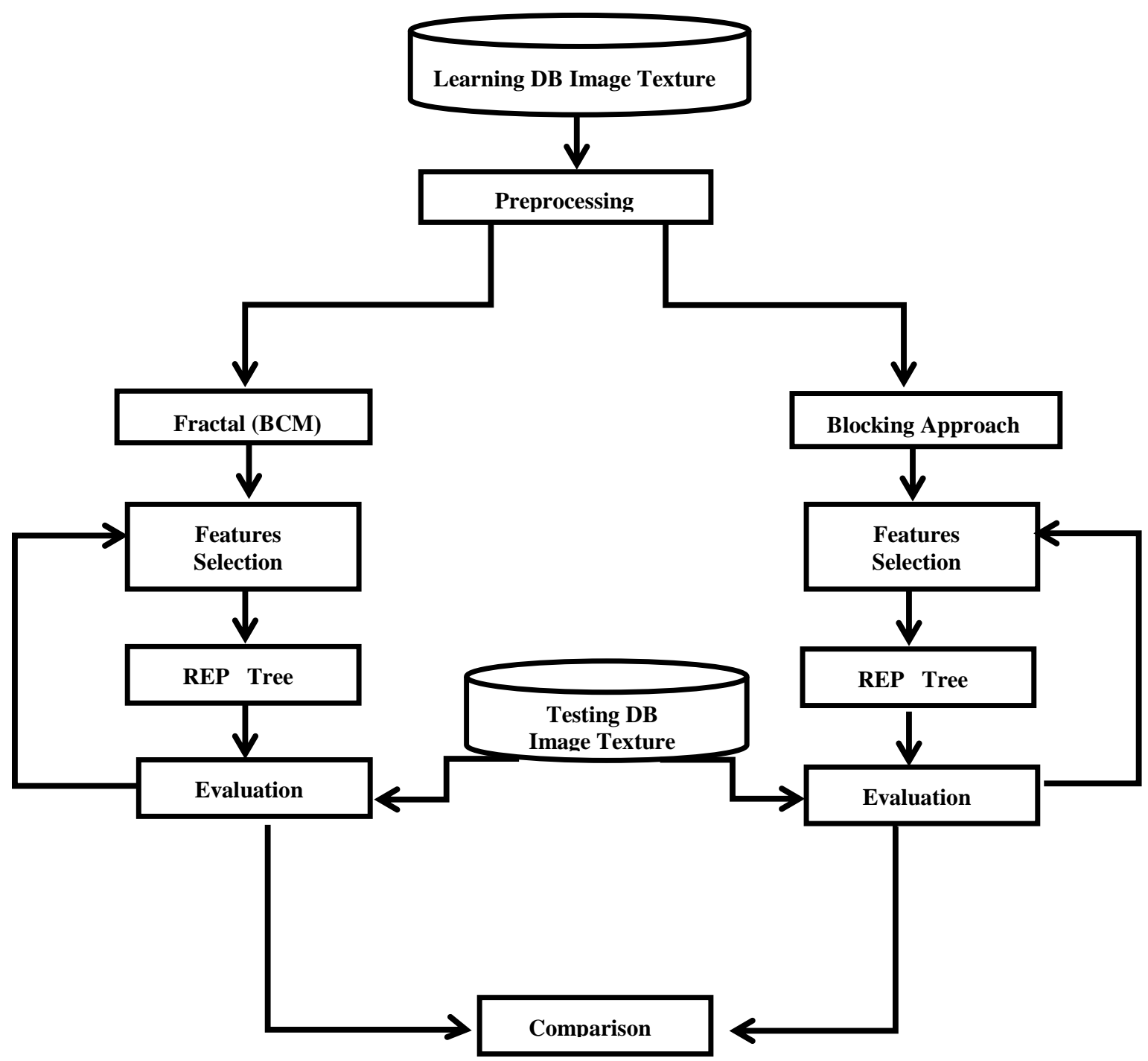

Figure (1): Block diagram of proposed system

\subsection{Learning Data Base Image Texture}

In this study, The samples chosen for studying and analyzing the textures' images in order to put the benefit relations will be too useful to describe other textures' images in advance step of this work. The database used for learning consists of 100 images. These image extensions are in JPG of their formats; the size of each learns image is $256 \times 256$ pixels.

\subsection{Pre-processing}

An application technique of suitable physical constraints to the observed scene may be used to significantly reduce the complexity of subsequent stage, called image equation ,that leads to Careful structuring of the lighting arrangement and camera position may be used to enhance the particular features of interest, Which results a good sample of image texture by converting process to gray level image. In color image : each pixel in the image consists of three principal colors (red, green, and blue). Each color component is represented by one byte. The gray value is obtained by applying the following equation:

$$
\text { Gray level value }=\frac{\operatorname{Re} d+\text { Green }+ \text { Blue }}{3}
$$

Finally, to reduce time complexity the gray image is resized by wavelet. Following algorithm (1) compute gray level and resizing by wavelet from image.

Algorithm 1: This algorithm shows sequence steps of compute gray level and resizing image. Input: Images texture with size NxN.

Output: Set of resized gray level image

1. Begin

2. For each inters image texture

3. Set image to gray level

4. Resize image in to (N/4) by wavelet

5. Save image

6. End

7. End

\subsection{Fractal Dimension with BCM}

In the algorithm 1 the pseudo-code of the proposed method is given by listing the main steps involved in calculating the fractal measure for image texture. The pseudo code reflects the "skeleton" in 3D space with $x-y$ axis as denoting $2 \mathrm{D}$ position and $\mathrm{z}$ coordinate as object appearance . By binarization methods gray-level image slides into several binary image Then in each binary image slide window, boxes with sizes $\mathrm{s}$, (where $\mathrm{s}=2 . .32, \mathrm{~W}$ mode $\mathrm{s}=0$ ). From all 
the $3 \mathrm{D}$ boxes, individual volume percentages are summed up to find the "count" $(\mathrm{N}(\mathrm{s}))$ value for each box size (s), $\mathrm{H}$ is slop, $\mathrm{A}(\mathrm{s})$ is total number of boxes to all block, $\mathrm{N}(\mathrm{s})$ is number of box cover the structure, $\mathrm{k}$ is highest box size and $\mathrm{s}$ is box size. The FD of a curve can be ranging between 1 (which is equal to its topological dimension) and 2 (which is equal to the topological dimension of the plane that it can occupy).

Algorithm 2: The box-counting algorithm for volume Percentage and modify Box-length.

Input: Image texture with size NxN.

Output: Feature Vector for each slide image

1. Begin

2. Sliding binary image by binarization method

3. Foreach slide of binary image do

4. Foreach Box size $\mathrm{s}$ from 2 to 32 where (W mode $\mathrm{s}=$ zero) of the Window do

5. $\mathrm{N}(\mathrm{s})=0$;

6. Foreach Non-overlapping Box in the slide do

7. Find the object box in $3 \mathrm{D}$ for the image surface (the "blanket");

8. volp $=$ percent of object appearance (the "blanket") in the box;

9. $\quad \mathrm{N}(\mathrm{s})=\mathrm{N}(\mathrm{s})+$ volp ;

10. End

11. End

12. End

13. For each binary slide image do

14. $\mathrm{H}=\frac{\sum_{s=0}^{k-2} \log (A(s)) * \log (N(s))}{\sum_{s=0}^{k-2} \log (A(s))^{\wedge}}$

15. $\mathrm{FD}=2-\mathrm{H}$

16. End

17. End

\subsection{Blocking Approach}

Feature extraction from binary image texture is done by statistical way. image texture consist in high order boxes each one contain 4 pixel then It assigned as (triple1 object, triple2 object, triple 3 object, triple 4 object, vertical1, vertical2, main diagonal object, secondary diagonal, horzentall object and horzental 2 object box) according to object distribution on these boxes. These boxes have ability to explain texture topology statistically.

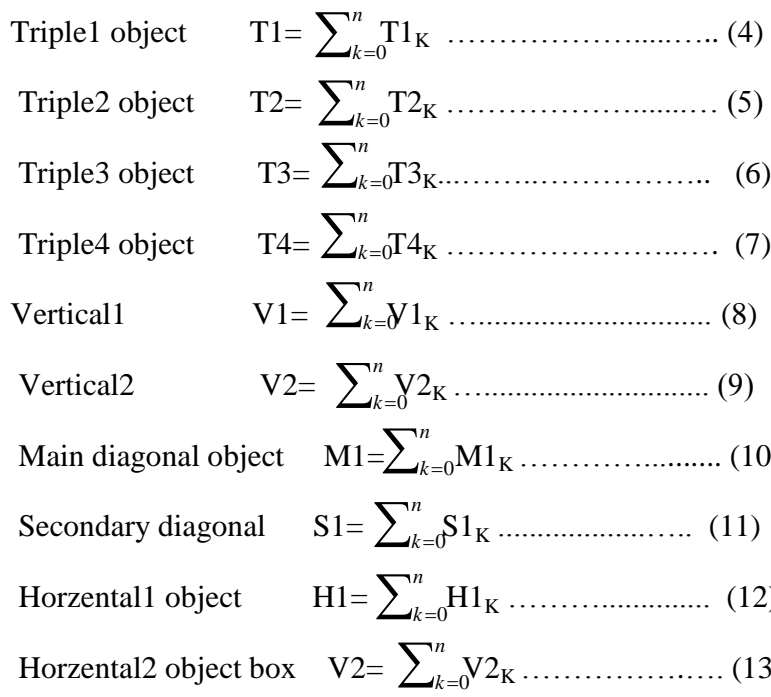

Algorithm 3: This algorithm shows analyzing steps of computing blocking approach. The output from this algorithm statistical indicator of image texture topology

Input: Image texture with size $\mathrm{NxN}$, Box-size [2,2].

Output: Feature Vector for each slide image

1. Begin

2. Set to binary image by $\mathrm{T}$ threshold

3. For each image size do

4. For each Non-overlapping Box in $\mathrm{W}$ do

5. Get Box

6. Compare with blocks model

7. Compute topology

8. End

9. End

10. End

\section{EXPERIMENTAL RESULT}

The evaluation result of classes are shown on Figures (2), (3) and (4), FD, BIA and Hybrid FD and BIA.

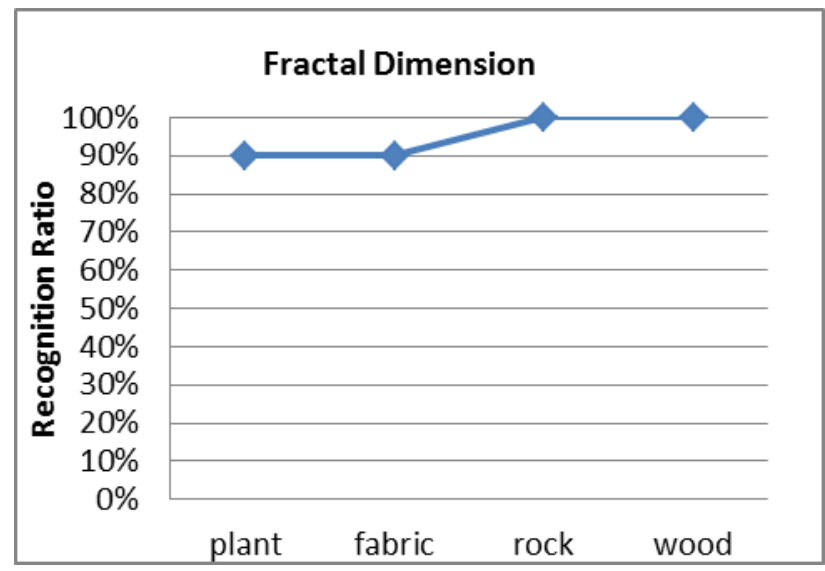

Figure (2) Recognition ratio of fractal dimension

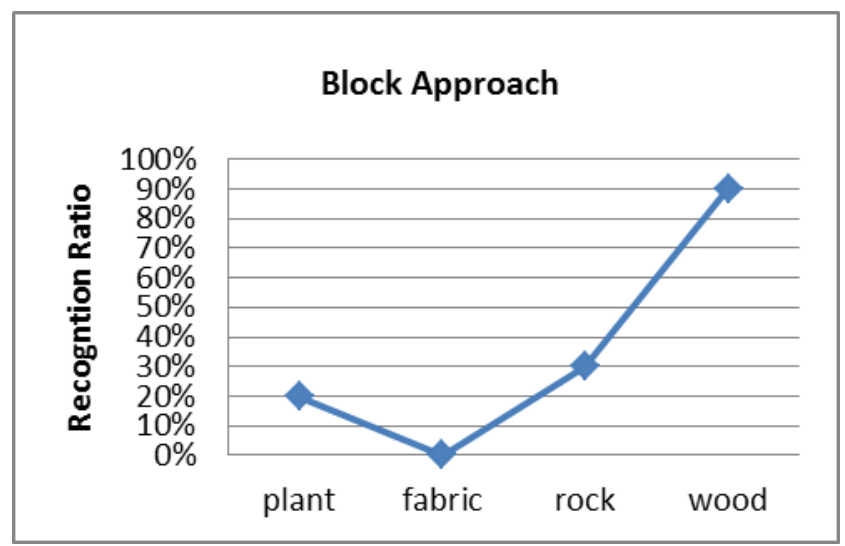

Figure (3) Recognition ratio of block Approach 


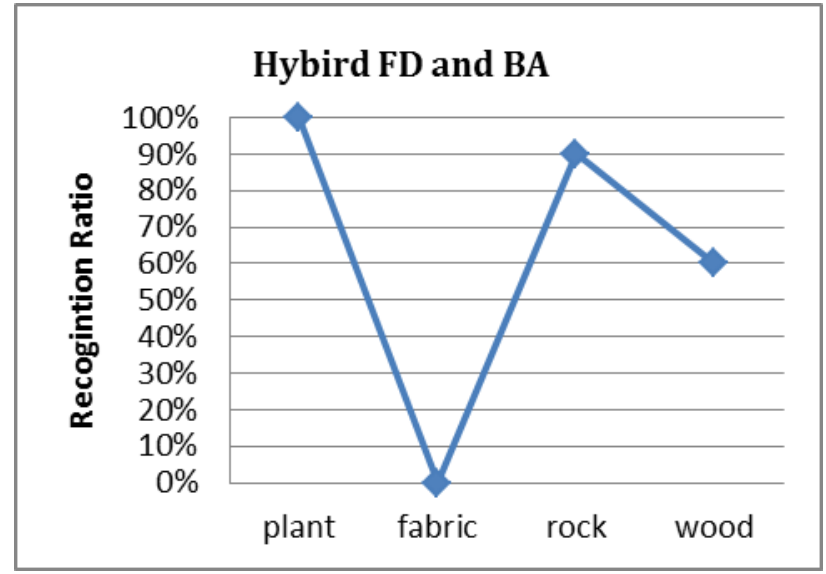

Figure (4) Recognition ratio of hybrid model

Previous figures show the results recognition of fractal dimension, block approach and the hybrid method of them which obtained a rate $95 \%, 40 \%$ and $65.5 \%$ respectively. At each method, discrimination ratios vary from Class to another, depending on the type of tissue in each Class. It records the highest percentage at class of wood in all previous methods because of the similarity of internal texture. The large differences in the composition of the different fabric texture and distribution of rocks lead to a decline in the proportion of discrimination by using block approach method. Whereas, hybrid method shows a little enhancement in the proportion of low discrimination in the block approach method. Because it depends on characteristics of both the fractal model and the block approach together.

\section{CONCLUCTION}

Three methods used to analyze and discriminate image texture of four different classes. The results of the highest discrimination ratio is $95 \%$ in fractal dimension whereas, $40 \%$ and $65.5 \%$ for block approach and hybrid methods respectively. The variation of Results refers to the different characteristics of each using method. Fractal dimension is more efficient in analyzing and discriminating in images textures.

\section{FUTURE WORK}

The present research suggests the following points for future work. These points associate with different applications of the fractal geometry and block approach in the recognition process of image.

$\checkmark$ Using color image texture technique to improve our approaches.

$\checkmark$ Comparing other measure for calculating fractal image as lacunarity with block approach.

$\checkmark$ Using other classification artificial intelligent technique as neural networks or other decision tree techniques.

\section{ACKNOWLEDGMENTS}

Special thanks would be towards all persons who have helped us in making this work see the light especially to Ahmed.T.Mahdi M.Sc. of engineering of Technology University .

\section{REFERENCES}

[1] Venus W.Samawe,"Investigation Into The Use of Neural Networks In Texture Classification", Ph.D.Thesis,Saddam University,Iraq,1999.
[2] Shaker Kadem Ali, " Texture Analysis and Classification by using Wavelet Transform and Neural Network", M.Sc.Thesis,University of Technology, Baghdad, 2003.

[3] Xianghua Xie ," A Review of Recent Advances in Surface Defect Detection using Texture Analysis Techniques", University of Wales Swansea,Electron Letters on Computer Vision and Image Analysis 7(3):122,2008 .

[4] B.Mandelbrot.The Fractal Geometry of Nature. W.H.Freeman and Company, New York, 1982.

[5] Rahib Abiyev and Kemal Ihsan Kilic," An Efficient Fractal Measure For Image Texture Recognition”, Dept. Of Computer Engineering, Near East University, Nicosia, Cyprus,2009.

[6] John C. Hart," Implicit Representations of Rough Surfaces", School of EECS Washington State University

[7] Saad Al-Shaban, Inaam A.M. Al -Sadik and Maha A. Amir," Blood Cells Images- Based on Chaos Theory", Communication and Electronics Department/ college of engineering, University of Jerash, Jerash, Jordan,2009.

[8] X.Xie,M.Mirmehdi, and B.Thomas,"Colour Tonality Inspection Using Eigenspace Features",Machine Vision and Applications , 2006.

[9] John C. Russ, "Theimage Processing Handbook" Third Edition, Materials Science and Engineering Department,North Carolina State University Raleigh, North Carolina, $(\mathcal{1} 1999$ by CRC Press LLC.

[10] Ling Guan, Yifeng He, Sun-Yuan Kung, "Multimedia Image and Video Processing" Second Edition, 2012 by Taylor \& Francis Group, LLC.

[11] Joao B. Florindo and Odemir M. Bruno," Texture analysis by multi-resolution fractal descriptors",2013

[12] Aazmit. Alrawi, Ali M.Sagheer and Dheyaa Ahmed Ibrahim, Texture Segmentation Based on Multifractal Dimension,2012.

[13] Akram Alsadat and Saeed Mozaffari, "Fractal and Multi-Fractal Dimensions For Farsi/Arabic Font Type and Size Recognition", Electrical and Computer Engineering Department, Semnan University, Iran, 2011.

[14] Ji-xiang Du, Chuan-Min Zhai and Qing-Ping Wang," Recognition of plant leaf image based on fractal dimension features", Huaqiao University, China,2012

[15] Losa G. A., Merlini D., Nonnenmacher T. F., Weibel E. (eds.), Fractals in Biology and Medicine, Vol. IV. Mathematics and Biosciences in Interaction. Birkhäuser Verlag, Basel, Switzerland. 2005.

[16] M. AI-Akaidi, "Fractal Speech Processing", Cambridge University press, 2004.

[17] Bourke., "An Introduction to Fractals", 1991.URL:http://www.local.wasp.uwa.edu.au/ pbourke/ fractals/fracintro.htm.

[18] T. Haghani, "Fractal Morphology \& Urban Complexity", phd thesis, School of Architecture, BIAD, Birmingham City University (BCU), UK, 2010. 